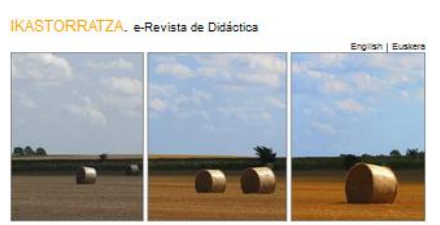

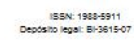

Inicio, Sobre nosotros, Publicacionesı, Participa

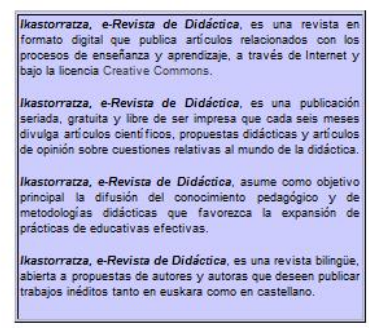

IKASTORRATZA. Didaktikarako e-aldizkaria

IKASTORRATZA. e-journal on Didactics

\author{
IRASTORRATZA. e-Revista de Didáctica
}

Acercamiento a la comprensión del concepto de ser vivo en educación infantil

A preliminary study concerning the understanding of the concept of living thing in early childhood

Olga Sanz Ortega

olgaso@bizkaia.eu

To cite this article:

Sanz, O. (2015). Acercamiento a la comprensión del concepto de ser vivo en educación infantil. IKASTORRATZA. e-Revista de Didáctica, 15, 99-118. DOI: 10.37261/15_alea/6

To link to this article:

http://www.ehu.es/ikastorratza/15_alea/ser_vivo.pdf

Published online: 20 Dic 2015. 


\title{
Acercamiento a la comprensión del concepto de ser vivo en educación infantil
}

\section{A preliminary study concerning the understanding of the concept of living thing in early childhood}

\author{
Olga Sanz Ortega \\ Graduada en Educación Infantil. UNIR \\ olgaso@bizkaia.eu
}

\section{Resumen}

Este trabajo pretende ser un acercamiento al estudio de la comprensión en torno al concepto de ser vivo en niños y niñas de entre 4 y 6 de edad. Para este fin se han aplicado varias pruebas a 82 escolares del segundo ciclo de educación infantil de manera individual. Estas pruebas han consistido en la realización de un test en torno al conocimiento de ser vivo y de dibujos relacionados con el mundo vegetal.

Los resultados de este trabajo indican que entre los 5 y 6 años se produce en los niños y niñas un avance significativo en lo relativo a la comprensión del concepto de ser vivo.

Los datos presentados sostienen la idea de que la capacidad para adquirir el conocimiento del concepto de ser vivo se desarrolla de manera constante durante la infancia, y así mismo, que este avance cognitivo viene influenciado por la experiencia física, social y cultural experimentada por el propio individuo.

Palabras clave: Educación Infantil, ser vivo, ente inerte, animismo, mundo vegetal

\begin{abstract}
This study presents a preliminary examination of how children from 4 to 6 begin to understand the essential notion of living thing. To that end, several tasks are proposed to a sample comprised of 82 children enrolled in the second level of Early Education. More specifically, the tasks used to carry out this study were a test related to the understanding of what a living thing is and, additionally, a drawing linked to plant world.

The results point out that the process of grasping the concept of living think develops significantly from 5 to 6 .

Moreover, the data suggests that the comprehension of the notion of living thing evolves constantly during the early childhood and, also, that this cognitive development in not alien to children's physical, social and cultural experiences that children go through.
\end{abstract}

Key words: Early Education, living thing, plants world, animism 


\section{Introducción}

Con intención de desarrollar el espacio teórico del estudio que nos ocupa, la revisión se centrará en profundizar dos planos bien diferenciados.

El primero se referirá a lo relacionado con la enseñanza de los aspectos relacionados a la comprensión de la noción de ser vivo dentro del marco normativo que lo atiende y lo desarrolla en el momento actual.

En segundo término, se presentará una revisión de lo estudiado en torno al desarrollo del concepto de ser vivo durante la etapa de la educación infantil. De esta manera, se podrá contextualizar y concretizar el estudio que nos ocupa.

\section{El concepto de ser vivo en el currículo de educación infantil}

El interés por la comprensión científica, el estudio del mundo natural y los elementos que lo integran desde edades tempranas, declarado en la literatura científica, es compartido por las instituciones educativas. Éstas lo concretan en la normativa que enmarca las actuales instrucciones formativas de los escolares de educación infantil.

De manera más específica, la comprensión de ser vivo y la correspondiente capacidad de diferenciarlos del mundo inerte y de los objetos no animados, es un asunto de gran transcendencia en el ámbito educativo y que está, asimismo, considerado en los niveles de educación infantil. Estos temas quedan evidenciados en los objetivos de la normativa educativa para la etapa de Educación Infantil (Orden ECI, 2008).

Dicha orden indica de manera textual en los objetivos para el segundo ciclo de infantil, que los escolares deberán adquirir, entre otras, la capacidad de:

... Interesarse por el medio natural, observar y reconocer animales, plantas, elementos y fenómenos de la naturaleza, experimentar, hablar sobre ellos y desarrollar actitudes de curiosidad... (Orden ECI, 2008, p. 1023).

En este sentido, se considera primordial que la escuela proporcione oportunidades para desarrollar un conocimiento científico relevante con relación al tema del conocimiento del entorno natural y biológico mediante la actividad educativa ajustada a cada edad y capacidades.

Se pretende, de esta manera, que los niños y niñas de infantil puedan construir esquemas de conocimiento en torno al mundo natural y los elementos que lo habitan más allá del conocimiento previo de cada individuo. Esta idea relativa a los conocimientos previos que los individuos tienen es un aspecto muy relevante en las etapas de Educación Infantil ya que pueden condicionar la aprehensión de nuevos conceptos. 
Investigadores como Garrido (2007), sostienen durante la etapa de Educación Infantil todavía no se comprenden los atributos biológicos que determinan a un ser vivo. Esto es así debido a que los niños y niñas de edad temprana centran su atención en el criterio del movimiento a la hora de decidir si un elemento es un ser vivo o no.

Por otro lado, la construcción durante la infancia de un conocimiento en torno a los fenómenos biológicos que de alguna manera se ajuste al propio conocimiento científico, promoverá la elaboración de una visión amplia del mundo natural que puede influir en la construcción de una conciencia vinculada a la conservación y cuidado del medio ambiente (Villarroel, 2013; Villarroel e Infante, 2014).

Este hecho en concreto es uno de los objetivos que queda reflejado en la normativa educativa para este ciclo en los contenidos establecidos:

[...] Conocer y valorar los componentes básicos del medio natural y algunas de sus relaciones, cambios y transformaciones, para desarrollar actitudes de cuidado, respeto y responsabilidad en su conservación [...] Orden ECI, 2008, p. 1023).

En cualquier caso, no es algo novedoso observar que el ser humano desde su infancia muestra gran interés y curiosidad por los elementos y fenómenos naturales que le rodean. Esta conducta exploratoria se ha vinculado con la "curiosidad natural" e "interés innato" hacia lo nuevo y diferente (Bijou, 1998). Este tipo de conductas desempeñan una función importante en el desarrollo de cada individuo.

Por medio de la interacción con el mundo natural, el ser humano puede construir las estructuras de conocimiento necesario para el propio desarrollo del individuo.

Es por esto que desde la normativa vigente (Orden ECI, 2008) se hace referencia a la siguiente idea que deberá atender el profesional de la enseñanza a la hora de realizar actuaciones educativas:

[...] El medio natural y los seres y elementos que lo integran son objeto preferente de la curiosidad e interés infantil. Las vivencias que tienen en relación con los elementos de la naturaleza y la reflexión guiada sobre ellas, les llevarán, con el apoyo adecuado de la escuela, a la observación de algunos fenómenos, sus manifestaciones y consecuencias, así como a acercarse gradualmente al conocimiento de los seres vivos, de las relaciones que se establecen entre ellos, de sus características y de algunas de sus funciones [...] (Orden ECI, 2008, p. 1023).

Otra idea a la que la misma normativa hace referencia es la relativa a la importancia de fomentar en los niños y niñas de infantil:

[...] La apreciación de la diversidad y riqueza del medio natural, el descubrimiento de que las personas formamos parte de ese medio, la vinculación afectiva al mismo, son la base para fomentar desde la escuela actitudes habituales de respeto y cuidado [...] (Orden ECI, 2008, p. 1023).

Lo que se pretende en definitiva, es lograr que el niño o niña de infantil a través del conocimiento del mundo natural logre desarrollar actitudes de respeto y cuidado. Estos valores son 
considerados necesarios para desarrollar una adecuada integración en el entorno escolar ya que durante los niveles iniciales de escolarización, los niños y niñas comienzan a asimilar actitudes de respeto hacia sus semejantes, hacia el entorno natural y sus integrantes.

Por consiguiente, el conocimiento del mundo natural deberá ser tanto un objetivo de enseñanza en Educación Infantil como un medio para obtener, entre otros fines, actitudes de cuidado, respeto y convivencia entre los más pequeños.

Por todas las anteriores cuestiones mencionadas, la comprensión de qué es un ser vivo, qué los caracteriza y qué los difiere de los entes no animados adquiere gran relevancia en ésta.

Por otro lado se debe hacer referencia y tener en cuenta a las diferentes comunidades autónomas, por tener estas la competencia de aplicar lo que se establece en la Ley Orgánica 2/2006, de 3 de mayo, de Educación para los diferentes niveles educativos.

A este respecto, en la comunidad autonómica del País Vasco , donde se ha realizado el estudio que se presenta, la antedicha ley orgánica se concreta en el Decreto 12/2009, de 20 de enero.

En la comunidad Vasca y con relación a la etapa de infantil, el mencionado reglamento establece entre los objetivos para el segundo ciclo de Educación Infantil aquellos relativos al ámbito de conocimiento del entorno. De esta manera, en el sexto de estos objetivos se señala lo siguiente:

[...] Observar y reconocer animales, plantas, elementos y fenómenos de la naturaleza, así como experimentar y hablar sobre ellos para interesarse por el medio natural y desarrollar actitudes de curiosidad y respeto [...]

Del mismo modo la segunda parte de este sexto objetivo para educación infantil indica que los escolares de esta etapa escolar deberán:

[...] Conocer y valorar los componentes básicos del medio natural y algunas de sus relaciones, cambios y transformaciones, para desarrollar actitudes de cuidado, respeto y responsabilidad en su conservación [...] (Decreto 12/2009, de 20 de enero, p. 22)

En definitiva, y tal como aparece reflejado en los contenidos de la normativa educativa actual para la Comunidad Autónoma Vasca, las instituciones educativas muestran gran interés en lo relativo a los temas relacionados con la comprensión del mundo natural en dos sentidos: como fuente de conocimiento del mundo físico y natural; y como medio para el conocimiento del propio ser humano y del individuo y del papel que este desempeña en la naturaleza.

\section{El desarrollo del concepto de ser vivo durante la infancia}

El concepto de ser vivo ha sido objeto de una amplia reflexión tanto desde la perspectiva científica y desde el ámbito de la educación científica (El-Hani, 2008), como desde la propia enseñanza-aprendizaje de este concepto (Adúriz-Bravo y Aymerich, 2005). Esta es una cuestión de indudable valor por poder favorecer en el individuo una comprensión amplia acerca del área del conocimiento relativo a la biología. 
En este sentido y centrándonos en la cuestión del estudio relativo a cómo se desarrolla la comprensión del concepto de ser vivo durante la infancia, éste resulta estar muy relacionado con dos corrientes de pensamiento relativas a la forma de entender el propio proceso de maduración cognitiva del ser humano.

Por un lado, se presentan aquellas perspectivas que se centran en analizar el papel del desarrollo y también de las limitaciones que durante el proceso de maduración cognitiva del individuo condicionan a éste con relación a la posibilidad de generar explicaciones sobre fenómenos biológicos, en general, y sobre la cuestión de qué es un ser vivo, en particular.

A diferencia de estas perspectivas destacan, por otro lado, aquellas líneas de estudio que se centran en el análisis del papel que juegan la experiencia física y cultural en el desarrollo de esta comprensión, más allá de las propias limitaciones evolutivas (Solomon \& Zaitchik, 2012).

La primera de las visiones mencionadas está estrechamente ligada a la tradición piagetana y de alguna manera sostiene la idea de este autor en lo concerniente al desarrollo del pensamiento animista durante la infancia (Piaget, 1929).

En este sentido, la perspectiva teórica de esta primera línea de estudio parte de las limitaciones que los niños pequeños presentan a la hora de manejar conceptos abstractos y comprender las relaciones causaefecto no evidentes que subyacerían a muchos fenómenos biológicos. También subraya la limitación que supone el característico egocentrismo ontogénico propio de la etapa pre-operacional. Esta etapa se relaciona con la limitación que presentan los niños de edad temprana relativa a los límites entre lo subjetivo y lo objetivo. Este obstáculo lleva a los niños pequeños a atribuir de manera habitual características de su mundo personal y subjetivo a los objetos reales.

De esta manera se explicaría la tendencia que los niños manifiestan en el sentido de transferir características propias de los seres vivos a los objetos inanimados (Solomon \& Zaitchik, 2012), especialmente a aquellos que muestran movimiento, como vehículos a motor y los fenómenos atmosféricos (veamos las nubes, coches, etc.). En este mismo sentido, se interpretaría también el hecho relativo a que los niños de edad temprana encuentran difícil distinguir como seres vivos aquellas entidades vivas de naturaleza inmóvil, como las plantas.

Consecuentemente, desde esta perspectiva de origen piagetano y por causa de las limitaciones que los niños pequeños pueden mostrar en cuanto al pensamiento lógico, es difícil aceptar que las creencias que aparecen durante la infancia relativas al concepto de ser vivo, especialmente hasta superar la etapa preoperacional, realmente puedan tener algún valor de coherencia interna o valor predictivo. De esta manera, difícilmente se acepta que las teorías explicativas que se desarrollan durante la infancia puedan presentar características comunes o, en alguna medida, puedan parecerse a aquellas desarrolladas a través del pensamiento adulto.

En las últimas décadas, sin embargo, se han llevado a cabo amplias y diversas investigaciones que han aportado nuevas perspectivas en torno al pensamiento infantil. Éstas, en cierta 
manera, desafían los presupuestos de las ideas piagetanas en torno al valor de las interpretaciones intuitivas que los niños pequeños realizan sobre el concepto de ser vivo.

La tesis que fundamenta estas líneas de investigación se relaciona con la idea de que el desarrollo conceptual está supeditado a ciertos aspectos del conocimiento e influenciado por la interacción de, por un lado, la capacidad cognitiva innata y común a todos los seres humanos (Spelke \& Kinzler, 2007), y por otro lado, la propia experiencia social y cultural con la que el sujeto se relaciona (Vigotsky, 1979).

A partir de estos dos factores, los individuos tienden a generar explicaciones espontáneas o teorías intuitivas que no necesariamente coinciden con las explicaciones científicas, pero en las que caben conceptos abstractos y explicaciones causales que se alejan de la simple observación de los fenómenos.

De esta manera, el desarrollo cognitivo no se entiende como un proceso madurativo de superación de fases de desarrollo, sino como un proceso de superación de teorías previas espontaneas hacia aquellas dotadas del necesario rigor científico.

Por consiguiente, la experiencia física y social jugaría un papel fundamental, no como propone la perspectiva piagetana de mero coadyuvador o, en su caso, limitador en el impulso de un modelo de desarrollo cognitivo universal, sino como elemento modelador esencial. Este hecho, unido a las interacciones con una capacidad cognitiva innata, marcaría la evolución de los conceptos y habilidades cognitivas que se desarrollarían durante la infancia.

En este sentido, y en concordancia con la línea teórica piagetana, autores como Delval (1999), consideran que las concepciones animistas que expresan los niños relativas a objetos inanimados se encuentran ligadas a conceptos como la fuerza y el movimiento, y que estas concepciones desaparecen según va avanzando el desarrollo intelectual.

Sin embargo según aporta Delval (1999), a través de sus trabajos en torno a conceptos científicos como la fuerza y calor entre niños y adolescentes de entre 4 y 20 años, estas concepciones intuitivas no solo no desaparecen durante la etapa pre-operacional, sino que se siguen manteniendo en etapas posteriores como la adolescencia. De ahí la importancia de promover una adecuada actividad escolar que cambie y desarrolle las concepciones innatas e intuitivas relativas a conceptos científicos.

En este sentido, existen numerosos estudios interesados en buscar respuestas a cómo favorecer en los escolares de infantil la comprensión en torno a la naturaleza de la ciencia y de sus conceptos (Adúriz-Bravo et al., 2005; Coll, 2005).

De este modo, autores como Gutiérrez (2004) apuntan a que la modelización puede ser una estrategia didáctica adecuada para fomentar el cambio conceptual en los niños y niñas. En este sentido, este autor considera necesario promover procesos de enseñanza-aprendizaje que ayuden en la formación de teorías científicas.

En tal sentido, el diseño de estrategias de enseñanza-aprendizaje relativas a la educación científica mediante el uso de modelos y analogías, sería la estrategia idónea para que los escolares de 
infantil construyesen modelos científicos precursores, y por ende, desarrollasen una progresiva comprensión acerca de la naturaleza de la ciencia y de sus conceptos (Adúriz-Bravo \& Aymerich, 2005).

Asimismo el enfoque de modelización (partiendo de la perspectiva de la ciencia escolar), ha mostrado evidencias de que los niños y niñas de edad temprana construyen el conocimiento a través del pensamiento, el lenguaje y la acción (Canedo, 2008; Canedo, Castelló, García, Gómez, y Morales, 2009, 2012)

Es tal vez que por todo lo anteriormente expuesto, las instituciones educativas toman en consideración muchas de las reflexiones en torno a la construcción de conocimiento relativo a la naturaleza y a sus elementos. Además proponen en las normativas que regulan las enseñanzas para la etapa de infantil, la utilización en las instrucciones educativas de metodologías activas donde el escolar sea protagonista de sus aprendizajes con el objeto de desarrollar dichas capacidades, tal y como se menciona en el Real Decreto 1630/2006, del 29 de diciembre al señalar lo siguiente:

[...] Observar y explorar de forma activa su entorno, generando interpretaciones sobre algunas situaciones y hechos significativos, y mostrando interés por su conocimiento [...] (p. 479) 


\section{Objetivos}

La investigación que se describe para este trabajo plantea realizar un estudio preliminar en torno al desarrollo del conocimiento del concepto de ser vivo entre niños y niñas desde 4 a 6 años de edad

Para este fin se proponen los siguientes cuatro objetivos:

1. Examinar el nivel de conocimiento en relación al concepto de ser vivo expresado por los niños y niñas de entre 4 y 6 años.

2. Analizar si los niños y niñas de entre 4 y 6 años pueden clasificar correctamente plantas y animales como seres vivos y, por otro lado, objetos móviles y entidades inertes como no- vivos.

3. Examinar el contenido de los dibujos que los niños y niñas realizan en torno al mundo vegetal para comprender el valor que la expresión gráfica infantil tiene en el desarrollo de la comprensión de conceptos relativos al medio ambiente.

4. Analizar la relación que las variables vinculadas con los objetivos previos (nivel de desarrollo del concepto de ser vivo y representación gráfica del mundo vegetal) presentan con el sexo y la edad de los individuos de la muestra.

El propósito final de este trabajo no es sino reunir evidencias que pudieran ayudar a la puesta en marcha de diseños didácticos que faciliten la comprensión en las etapas de Educación Infantil de las plantas como seres vivos. 


\section{Método}

\section{Muestra}

La investigación que se plantea en este trabajo está dirigida hacia niños y niñas del segundo ciclo de Educación Infantil. De esta manera se propone que la muestra a estudiar esté compuesta en torno a unos 80 niños y niñas divididos en dos grupos de edad: 4-5 (nacidos en 2010) y 5-6 años (nacidos en 2009).

Se proyecta desarrollar el trabajo en el colegio público de Gorliz, localizado en el municipio vizcaíno del mismo nombre.

\section{Procedimiento}

El procedimiento previsto para esta investigación se basa en la entrevista con los niños y niñas y en el estudio de los dibujos que estos lleven a cabo en torno a la cuestión de las plantas y la vida vegetal.

El procedimiento previsto se detalla a continuación:

a.) Presentación de la actividad en el aula

En primer lugar, se presentará, con la ayuda de la profesora en el aula, tanto la actividad como la persona encargada del estudio y explicará al alumnado quién es y qué se propone hacer en las próximas sesiones.

A continuación y con el fin de llevar a cabo la actividad, se relatará la historia sobre un pequeño animal que vivía en el desierto; en un entorno natural árido, rodeado de dunas y grandes extensiones de arena. Lo peculiar de esta narración es que el pequeño personaje de esta historia descubre que no conoce nada sobre las plantas y el mundo vegetal y pretende cambiar esta situación. El relato se encuentra en el anexo, al final del trabajo.

A este respecto, se llevará al aula un muñeco que será la representación tangible del personaje de la historia contada con anterioridad (un pequeño dromedario, que en todo momento se llamará "Pequeño Drome").

Los siguientes dos pasos se llevarán a cabo de forma individualizada con cada uno de los participantes en el estudio en un rincón apropiado dentro la misma aula o lo más cercano a la misma. 
b.) Dibujo en torno a las plantas

En la entrevista individual se propone al escolar que realice un dibujo para mostrar al protagonista del cuento qué son las plantas. El muñeco que representa al protagonista de la narración, estará presente en la entrevista y debe servir como recurso para motivar al niño o niña en la realización del dibujo.

Del mismo modo, se le pedirá a cada escolar que represente todos aquellos elementos que conozca relacionados con el mundo vegetal y las plantas, con el objeto de explicárselos al muñeco.

Una vez acabado el dibujo, se repasará con cada niño o niña los elementos dibujados para dejar constancia del significado de cada uno de ellos en el reverso del dibujo.

c.) Test de conocimiento sobre el concepto de ser vivo

Una vez el escolar haya realizado su representación gráfica, se pasará a la siguiente fase de la entrevista. Ésta consistirá en realizar a cada escolar de manera individual un test relacionado con el concepto de ser vivo.

Con este fin se presentarán de una en una, 8 fotografías de los siguientes seres vivos o entidades inertes: un pájaro, el sol, varias piedras, las nubes, una flor, una moto, un árbol y un perro). Con cada fotografía se preguntará a cada escolar en torno a si lo que se muestra en la foto es o no, un ser vivo.

Al termino de la recogida de los datos referentes a cada aula los diferentes elementos dibujados por los escolares se clasificaran en cinco categorías; elementos vegetales (plantas, hojas, árboles, raíces, flores, hierba y semillas); otros seres vivos (animales y mascotas); agentes atmosféricos (nubes, sol, cielo, arco iris, lluvia, estrellas); elementos del entorno (montes, suelo, recipientes de las plantas, ríos); otros elementos (corazones, monstruos, pelotas y vehículos).

d.) Tratamiento de datos

De cada participante se recogerán los siguientes datos:

a.) Personales: Nombre, mes y año de nacimiento, curso escolar y sexo.

Con relación al dibujo:

b.) frecuencia de elementos vegetales dibujados: plantas completas, hojas, árboles, raíces, flores, hierba y semillas.

c.) frecuencia de otros seres vivos dibujados: animales y mascotas.

d.) frecuencia de elementos dibujados relacionados con los agentes atmosféricos: nubes, sol, cielo, arco iris, lluvia, estrellas.

e.) frecuencia de elementos dibujados relacionados con el entorno: montes, suelo, recipientes de las plantas, ríos.

f.) otros elementos: corazones, monstruos, pelotas y vehículos. 
Con relación a la entrevista:

g.) frecuencia de errores en las imágenes de los seres vivos

h.) frecuencia de errores en las imágenes de las entidades inertes.

\section{Resultados}

A continuación se presentan los resultados del estudio realizado. En primer lugar se dará cuenta de las características de la muestra en su conjunto. Posteriormente se presentará el análisis de las diferencias que existen entre los dos grupos de edad considerados (nacidos en el 2009 y nacidos en el 2010) tanto con relación al test relativo al conocimiento del ser vivo como con relación al contenido de los dibujos llevados a cabo sobre el mundo vegetal. Finalmente se presentaran los resultados del examen de las diferencias entre niños y niñas con relación a las mismas pruebas.

\section{Con respecto a la muestra}

La muestra estudiada en este trabajo está compuesta por 82 participantes, de los cuales 39 son niñas y 43 son niños.

La edad de los escolares es de entre 4 y 6 años. En este trabajo se han considerado dos grupos de edad: los nacidos en el 2009 ( $2^{\circ}$ curso de segundo ciclo de educación Infantil) y los nacidos en el 2010 ( $1^{\circ}$ curso de segundo ciclo de educación Infantil).

En la Figura 1 se presenta la distribución de la muestra con relación a la edad.

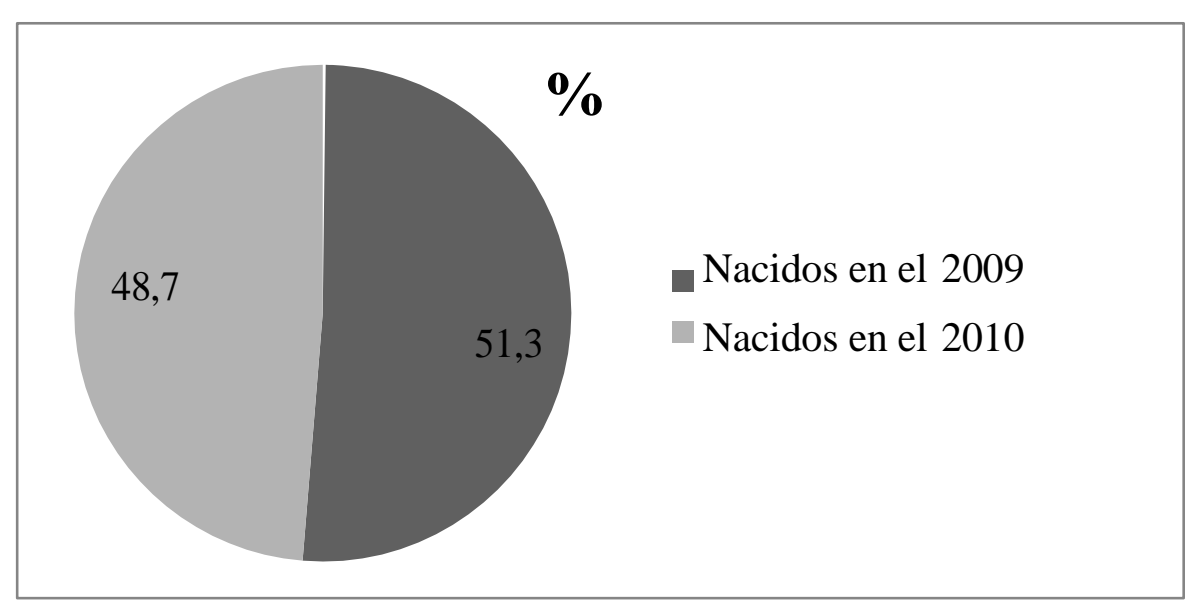

Figura 1: Representación del porcentaje de estudiantes en cada grupo de edad considerado en el estudio 


\section{Con respecto a las diferencias relacionadas con la edad}

a.) Test de conocimiento del ser vivo

De los 82 escolares que participaron en este estudio, solamente 2 de todos ellos lograron responder correctamente a todas las preguntas del test relacionado con el conocimiento del concepto de ser vivo.

En la Figura 2 se presenta el porcentaje de respuestas correctas en cada una de las entidades consideradas en el test y para cada uno de los grupos de edad estudiados.

\begin{tabular}{c|ccccc|}
\multicolumn{2}{c}{ Animales } & Plantas & Vehículos & Atmósfera & Piedras \\
\hline 2009 & 85,7 & 35,7 & 66,7 & 45,2 & 61,9 \\
$(\mathrm{~N}=42)$ & & & & & \\
\hline 2010 & 66,7 & 52,5 & 37,5 & 40 & 42,5 \\
$(\mathrm{~N}=40)$ & & & & & \\
\hline
\end{tabular}

Figura 2: Frecuencia relativa (\%) de respuestas correctas en el test de conocimiento del concepto de ser vivo para cada una de las entidades estudiadas.

Cuando se analizan las diferencias en cada categoría entre los dos grupos de edad, éstas resultan significativas únicamente en el caso de la categoría de vehículos (Chi-Square $=6,9[1] ; p<0,01)$.

En la Figura 3 se presenta el porcentaje de respuestas correctas e incorrectas para el caso de la categoría de vehículos.

\begin{tabular}{c|c|c|}
\multicolumn{2}{|c|}{ incorrecto } & correcto \\
\hline $2009(N=42)$ & 33,3 & 66,7 \\
$2010(N=40)$ & 62,5 & 37,5 \\
\hline
\end{tabular}

Figura 3: Frecuencia relativa de respuestas correctas e incorrectas (\%) en la categoría de "vehículos" del test de conocimiento del concepto de ser vivo considerando el año de nacimiento de los participantes de la muestra. 
b.) Análisis del contenido de los dibujos

En conjunto los niños y niñas de la muestra estudiada dibujaron un total de 257 elementos. Los elementos pictóricos encontrados en los dibujos fueron clasificados en 5 categorías, las cuales que se presentan en la Figura 4.

\begin{tabular}{|c|c|c|c|c|c|}
\hline $\begin{array}{l}\text { Categoría } \\
\text { considerada }\end{array}$ & $\begin{array}{l}\text { Elementos } \\
\text { vegetales }\end{array}$ & $\begin{array}{l}\text { Otros seres } \\
\text { vivos }\end{array}$ & $\begin{array}{c}\text { Agentes } \\
\text { atmosféricos }\end{array}$ & $\begin{array}{l}\text { Elementos } \\
\text { del entorno }\end{array}$ & $\begin{array}{c}\text { Otros } \\
\text { elementos }\end{array}$ \\
\hline $\begin{array}{l}\text { Tipo de } \\
\text { elemento } \\
\text { pictórico }\end{array}$ & $\begin{array}{c}\text { Plantas, } \\
\text { hojas, árboles, } \\
\text { raíces, flores, } \\
\text { hierba y } \\
\text { semillas }\end{array}$ & $\begin{array}{c}\text { Personas, } \\
\text { animales y } \\
\text { mascotas }\end{array}$ & $\begin{array}{l}\text { Nubes, sol, } \\
\text { cielo, arco } \\
\text { iris, lluvia, } \\
\text { estrellas }\end{array}$ & $\begin{array}{c}\text { Montes, } \\
\text { suelo, } \\
\text { recipientes } \\
\text { de las } \\
\text { plantas, ríos }\end{array}$ & $\begin{array}{c}\text { Corazones, } \\
\text { monstruos, } \\
\text { pelotas y } \\
\text { vehículos }\end{array}$ \\
\hline
\end{tabular}

Figura 4: Tipo de elementos pictóricos considerados en las cinco categorías del estudio

Por otro lado, en la Figura 5 se presentan las frecuencias con las que los elementos pictóricos de cada categoría aparecen en cada uno de los dos grupos de edad considerados. Estas diferencias son significativas (Chi-Square $=11,5[4] ; \mathrm{p}<0,05)$.

\begin{tabular}{c|c|c|c|c|c|}
\multicolumn{2}{c|}{$\begin{array}{c}\text { Elementos } \\
\text { vegetales }\end{array}$} & $\begin{array}{c}\text { Otros } \\
\text { seres vivos }\end{array}$ & $\begin{array}{c}\text { Agentes } \\
\text { atmosféricos }\end{array}$ & $\begin{array}{c}\text { Elementos } \\
\text { del entorno }\end{array}$ & $\begin{array}{c}\text { Otros } \\
\text { elementos }\end{array}$ \\
\hline $2009(N=133)$ & 49,6 & 15,8 & 8,3 & 17,3 & 9 \\
\hline $2010(N=124)$ & 41,1 & 19,3 & 6,5 & 10,5 & 22,6 \\
\hline
\end{tabular}

Figura 5: Frecuencia relativa (\%) de elementos diferentes que los escolares de la muestra estudiada dibujan en torno al mundo vegetal, atendiendo al año de nacimiento

Para realizar estas pruebas se ha empleado el programa Chi-Square a través de la web: http://www.quantpsy.org/chisq/chisq.htm 


\section{Con respecto a las diferencias relacionadas con el género}

a.) Test de conocimiento del ser vivo

En Figura 6 se muestra la frecuencia de contestaciones correctas que, por un lado, los niños y, por otro lado, las niñas lograron con relación a las preguntas de cada una de las entidades consideradas en el test. Las diferencias que se muestran no son significativas.

\begin{tabular}{|c|c|c|c|c|c|}
\hline & Animales & Plantas & Vehículos & Atmósfera & Piedras \\
\hline $\begin{array}{c}\text { Niñas } \\
(N=39)\end{array}$ & 74,4 & 43,6 & 56,4 & 43,6 & 53,8 \\
\hline $\begin{array}{l}\text { Niños } \\
(N=43)\end{array}$ & 81,4 & 44,2 & 48,8 & 41,9 & 51,2 \\
\hline
\end{tabular}

Figura 6: Frecuencia relativa (\%) de respuestas correctas en el test de conocimiento del concepto de ser vivo para considerando la variable género

b.) Análisis del contenido de los dibujos

Finalmente, en la Figura 7 se presentan las frecuencias con las que las niñas y los niños dibujaron elementos pictóricos en cada categoría. Las diferencias presentadas no son significativas.

\begin{tabular}{c|c|c|c|c|c|} 
& $\begin{array}{c}\text { Elementos } \\
\text { vegetales }\end{array}$ & $\begin{array}{c}\text { Otros seres } \\
\text { vivos }\end{array}$ & $\begin{array}{c}\text { Agentes } \\
\text { atmosféricos }\end{array}$ & $\begin{array}{c}\text { Elementos } \\
\text { del entorno }\end{array}$ & $\begin{array}{c}\text { Otros } \\
\text { elementos }\end{array}$ \\
\cline { 2 - 6 } Niñas $(N=132)$ & 44 & 19,7 & 8,3 & 15,9 & 12,1 \\
\hline Niños $(N=125)$ & 47,2 & 15,2 & 6,4 & 12 & 19,2 \\
\hline
\end{tabular}

Figura 7: Frecuencia relativa (\%) de elementos diferentes que los escolares de la muestra estudiada dibujan en torno al mundo vegetal considerando la variable género 


\section{Discusión de resultados}

Los resultados presentados indican, en primer lugar, que la edad resulta ser un factor ligado a las diferencias encontradas tanto en lo relativo a la capacidad de realizar correctamente el test sobre el concepto de ser vivo como en lo referente a los contenidos realizados sobre la vida vegetal.

Sin embargo, resulta también interesante que no se hayan encontrado diferencias significativas en ambos tipos de pruebas entre niños y niñas.

Considerando más en detalle las diferencias encontradas entre los dos grupos de edad con relación al test vinculado al conocimiento del ser vivo, en primer lugar hay que destacar que estas diferencias se vinculan a la mayor frecuencia de respuestas correctas con relación a la pregunta de si los vehículos a motor, más concretamente, las motos son o no seres vivos. Los datos presentados indican que los niños más mayores consideran más frecuentemente que efectivamente, las motos no son seres vivos.

Por otro lado, resulta llamativo que el resto de categorías no arrojen diferencias significativas entre ambos grupos de edad, indicando que las respuestas correctas a la cuestión de si los vegetales, los animales, el sol, las nubes y las piedras son o no seres vivos son igualmente frecuentes en ambos grupos de edad.

Finalmente los dibujos indican que los niños y niñas más mayores dibujan determinados elementos pictóricos más frecuentemente que los sus compañeros de menor edad. Atendiendo a los resultados presentados en la tabla 4, las mayores diferencias se centran en las categorías de Elementos vegetales, Elementos del entorno y Otros elementos.

Más concretamente, los niños y niñas más mayores dibujan más frecuentemente aspectos relacionados con Elementos vegetales y Elementos del entorno mientras que dibujan con menor frecuencia Otros elementos. De hecho la mayor diferencia entre los elementos dibujados por niños y niñas nacidos en el 2009 y aquellos nacidos en el 2010 se centra precisamente, en la categoría de Otros elementos, que está vinculada al dibujo de aspectos como "monstruos", "pelotas", "corazones" y "coches".

En conjunto, todas las consideraciones y datos presentados parecen resultar coherentes con el supuesto de que los niños y niñas nacidos en el 2009 han avanzado, aunque tímidamente, en lo relativo al conocimiento de qué es un ser vivo y que este avance se podría relacionar con hecho de ser más conscientes de que no todos los entes que se mueven estén vivos (como resultaría en el caso de los vehículos a motor; por ejemplo, las motos).

Por otro lado, el hecho de que en los dibujos de los niños y niñas más mayores 
aparezcan más frecuentemente elementos relativos a las plantas (hojas, raíces, semillas, árboles, hierba...) y con menor frecuencia otros elementos pictóricos, que nada tienen que ver con el mundo vegetal y natural, podría considerarse que los niños y niñas mayores han podido desarrollar un avance en el conocimiento del mundo vegetal y del concepto de ser vivo, a pesar de que este progreso todavía no se haya reflejado en una mayor frecuencia de la correcta clasificación de las plantas como seres vivos.

\section{Limitaciones de la investigación}

Los datos presentados sugieren líneas de trabajo ligadas a las propias limitaciones que la investigación llevada a cabo tiene.

En primer lugar hay que mencionar que sería necesario aumentar la muestra de forma que las conclusiones que se puedan extraer resulten ser más sólidas. Con un tamaño de muestra mayor los resultados serían mucho más concluyentes y significativos. Una posible vía de trabajo futuro podría estar relacionada, precisamente, con un aumento de la muestra empleada. Además también hay que considerar que la muestra no ha sido elegida al azar sino que por el contrario, ésta ha sido elegida por la responsable del trabajo. Este aspecto puede indudablemente, introducir un sesgo significativo. En consecuencia, trabajos futuros deberán tener en cuenta muestreos en colegios diferentes o muestras suficientemente amplias de centros educativos distintos.

Por otro lado, el trabajo presentado no considera otro tipo de variables que pueden debilitar la solidez de las conclusiones finales. Concretamente, no se ha considerado la posibilidad de que otro tipo de variables como, por ejemplo, la ubicación y tipología del centro y las características del entorno (rural, de núcleo urbano u otros) pueda influir en el desarrollo de la comprensión del concepto de ser vivo de los niños y niñas. Ésta puede ser una línea de trabajo interesante de cara al futuro.

Finalmente, y con relación a la propia metodología empleada, existen algunos aspectos que debieran mejorarse en trabajos futuros. Por un lado, las entrevistas con los participantes han sido realizadas por una única persona, de manera que los datos solo han sido codificados por la misma. En el caso de participar más de un entrevistador y evaluador se podrían establecer comparaciones entre éstos con el fin de valorar si se han utilizado criterios de codificación idénticos. Otras cuestiones relevantes pueden ser el hecho de utilizar fotografías para valorar el conocimiento de ser vivo que tiene los niños y niñas de Educación Infantil. Cabe preguntarse si las respuestas podrían variar en el caso de utilizar otros recursos (una posibilidad son ejemplares reales de los seres vivos). 


\section{Conclusiones}

¿Comprenden los niños y niñas de entre 4 y 6 qué es un ser vivo? Ésta resulta ser la pregunta inicial que ha guiado el desarrollo de este trabajo y lo cierto es que intentar responder a la cuestión a través de los datos presentados en este trabajo sería complicado.

No obstante, podemos afirmar que los resultados y datos mostrados en este trabajo son congruentes con la idea, defendida también por otros autores, de que durante el periodo que abarca el segundo ciclo de Educación Infantil los niños y niñas realmente manifiestan un nivel de comprensión incipiente relativo a qué es un ser vivo. Una de las aportaciones que este estudio preliminar presenta en línea con esta afirmación sería las diferencias observadas entre los dos grupos de diferente edad.

Estas diferencias se manifestarían especialmente en dos aspectos. Por un lado el alumnado de mayor edad clasifica más correctamente como entidades inertes a los vehículos. Este dato indicaría que ciertamente este alumnado ha dejado de relacionar el concepto de ser vivo con la noción de movilidad, aceptando que no todo lo que se mueve es un ser vivo. Igualmente se puede suponer que este grupo ha comenzado a entender que determinadas entidades, aparentemente inmóviles, comparten las características de la vida con otros seres vivos dotados de movimiento.

Por otro lado, los dibujos de los niños y niñas mayores presentan significativas diferencias con aquellos realizados por sus compañeros más jóvenes, sobre todo en lo relativo a elementos pictóricos que representan elementos esenciales para la vida vegetal, tales como el sol, la lluvia, las nubes.

De manera que a la vista de estas consideraciones, se podría entender que a estas edades los niños y niñas comienzan a aprehender aspectos esenciales del concepto de ser vivo especialmente relacionado con dos aspectos: (a) desvincular dicho concepto con la idea de movimiento y (b) entender el conjunto de necesidades y de características esenciales del mundo vegetal. A este respecto no se puede dejar pasar por alto, referenciado en (Villarroel e Infante, 2014) que la comprensión de las plantas como seres vivos es un significativo paso conceptual que los niños y niñas pequeños deben superar en su desarrollo de la comprensión de conceptos biológicos.

Estas ideas parecen contrastar con la perspectiva piagetiana del desarrollo conceptual, en la medida que parece que se acepta el hecho de que antes de la superación del estadio preoperatorio realmente ya se podrían identificar los primeros indicios conceptuales 
concernientes a nociones de significativo calado científico, como en este caso sería la comprensión del concepto de ser vivo.

Puede entenderse que las consideraciones antes dichas implican interesantes consecuencias educativas, ya que si entendemos que las limitaciones que la edad impone al desarrollo conceptual dejan margen al desarrollo de los primeros esbozos de la comprensión de conceptos científicos (en este caso vinculados a la noción de ser vivo), el profesorado entiende que es necesario explorar y llevar a cabo prácticas educativas que puedan estimular el desarrollo de estos conceptos incipientes que ya se inician en la mente de los niños y niñas pequeños.

En otras palabras, las limitaciones relativas al pensamiento formal que caracteriza al alumnado de la etapa escolar de Educación Infantil no serían justificación para no suponer que los niños y niñas pueden avanzar en la compresión del concepto de ser vivo, siempre y cuando se adoptaran las medidas y recursos educativos adecuados a su propio desarrollo. 


\section{Referencias}

Adúriz-Bravo, A., \& Aymerich, M. M. I. (2009). Un modelo de modelo científico para la enseñanza de las ciencias naturales. Revista electrónica de investigación en educación en ciencias, 4(1), 40-49.

Bijou, S. W. (1998). Conducta exploratoria en la infancia y en la niñez temprana. Revista Mexicana de Análisis de la Conducta, 1(2), 215-223.

Canedo, S. (2008). Contribución al conocimiento del estudio del aprendizaje de las ciencias experimentales en la educación infantil: cambio conceptual y construcción de modelos científicos precursores. Tesis Doctoral. Universidad de Barcelona. http://www.tdx. catlhandle/10803/1321.

Canedo, S., Castelló, J., García, P., Gómez, A. y Morales, A. (2009). Estudio del proceso de cambio conceptual y la construcción del modelo científico precursor de ser vivo en niños de pre-escolar. Enseñanza de las Ciencias, Número Extra VIII Congreso Internacional sobre Investigación en Didáctica de las Ciencias, Barcelona, pp. 2563-2568.

Canedo, S., Castelló, J., García, P., Gómez, A. y Morales, A. (2012). Cambio conceptual y construcción de modelos científicos precursores en educación infantil. Revista Mexicana de Investigación Educativa, 17(54), 691-727.

Delval, J. (1975). El animismo y el pensamiento infantil. Madrid: Siglo XXI.

El-Hani, C.N. (2008). Theory-based approaches to the concept of life. Journal of Biological Education, 42(4), 147-149.

Garrido, M. (2007). La evolución de las ideas de los niños sobre los seres vivos. (Tesis doctoral). Universidad da Coruña. Recuperado de: Repositório da UDC (http://hdl.handle.net/2183/7330).

Gutiérrez, R. (2004). La modelización y los procesos de enseñanza/aprendizaje. Alambique, 42, 8-18

Piaget, J. (1929).The Child's Conception of the World. New York: HarcourtBrace.

Snaddon, J.L., Turner, E.C., Foster, W.A. (2008) Children's Perceptions of Rainforest Biodiversity:Which Animals Have the Lion's Share of Environmental Awareness? PLoS ONE 3(7): e2579.doi:10.1371/journal.pone.0002579.

Solomon, G. \& Zaitchik, D. (2012). Folkbiology. Wiley Interdisciplinary Reviews: Cognitive Science, 3(1), 105-115.

Spelke, E. S., \& Kinzler, K. D. (2007). Core knowledge. Developmental Science, $10(1), 89-96$.

Vigotsky, L. S. (1979). El desarrollo de las funciones psicológicas superiores. Barcelona: Grijalvo. 
Villarroel, J. D. (2013). Environmental judgment in early childhood and its relationship with the understanding of the concept of living beings. SpringerPlus, 2(1), 1-13.

Villarroel, J.D. \& Infante, G. (2014). Early understanding of the concept of living things: An examination of young children's drawings of plant life. Journal of Biological Education, 48(3), 119-126.

\section{Legislación}

Decreto 175/2007, de 16 de octubre, por el que se establece el currículo de la Educación Básica y se implanta en la Comunidad Autónoma del País Vasco. (BOPV núm. 218 de 13 de noviembre de 2007).

Ley Orgánica 2/2006, de 3 de mayo, de Educación. (BOE núm 106 de 4 de mayo de 2006).

Orden 3960/2007, de 19 de diciembre, por la que se establece el Currículum de Educación Infantil y se regula la ordenación de la educación infantil. (BOE núm. 5 de 5 de enero de 2008).

Real Decreto 1630/2006, de 29 de diciembre, por el que se establecen las enseñanzas mínimas del segundo ciclo de Educación infantil. ( BOE núm. 4 de 4 de enero de 2007). 